PACS 31.15.A-

Kuznetsova A. A., Glushkov A. V., Plisetskaya E. K.

National University “Odesa Maritime Academy”, Didrikhson str. 8, Odesa, Ukraine

Odesa State Environmental University, 15, Lvovskaya str., Odesa-16, 65016, Ukraine

E-mail: kuznetsovaa232@gmail.com

\title{
THEORETICAL COMPLEX ENERGIES OF STARK RESONANCES IN LITHIUM BY OPERATOR PERTURBATION THEORY APPROACH
}

The theoretical complex energies of the Stark resonances in the lithium atom (non-hydrogenic atomic system) in a DC electric are calculated within the operator form of the modified perturbation theory for the non- $\mathrm{H}$ atomic systems. The method includes the physically reasonable distorted-waves approximation in the frame of the formally exact quantum-mechanical procedure. The calculated Stark resonances energies and widths in the lithium atom are calculated and compared with results of calculations on the basis of the method of complex eigenvalue Schrödinger equation by Themelis-Nicolaides, the complex absorbing potential method by Sahoo-Ho and the B-spline-based coordinate rotation method approach by Hui-Yan Meng et al.

\section{Introduction}

The Stark effect is one of the best known problems in quantum mechanics, however at the same time one of the most difficult in a case of the strong field one [1-8]. In the last years it attracts a great interest especially outside the weak-field region that is stimulated by a whole range of interesting phenomena to be studied such as the effects of potential barriers (shape resonances), new kinds of resonances above threshold etc [11-42], the DC strong field effect in the heavy atomic systems etc. The great relevance of the Stark resonances characteristics of the multielectron atoms is usually provided by standard requirements in spectroscopic information of a number of physical applications, which include atomic and molecular optics and spectroscopy, quantum electronics, laser physics, quantum computing, the construction of kinetic models of new laser schemes for shortrange, physics and chemistry laboratory, astrophysical plasmas, astrophysics and astronomy etc. An external electric field shifts and broadens the bound state levels of an atomic system. The standard quantum-mechanical procedure relates the complex eigenenergies (EE) $E=E_{r}+i \Gamma / 2$ and complex eigenfunctions (EF) to the shape resonances. The field effects drastically increase upon going from one excited level to another. The highest lev- els overlap forming a "new continuum" with lowered boundary. The computational difficulties (for example, such as the well-known Dyson phenomenon) inherent to the standard quantum mechanical methods are well known. The well-known Wentzel-KramersBrillouin (WKB) approximation overcomes these difficulties for the states lying far from the " new continuum" boundary. Some modifications of the WKB method (see review in Ref. [58]) are introduced in Stebbings and Dunning, Kondratovich and Ostrovsky, Popov et al; Ivanov-Letokhov ( e.g. citations in refs. [1-42]) have fulfilled the first estimations of the effectiviness of the selective ionization of the Rydberg atom using a DC electric and laser fields within the quasiclassical model.

Different computational procedures are used in the Pade and then Borel summation of the divergent Rayleigh-Schrödinger perturbation theory (PT) series (Franceschini et al 1985, Popov et al 1990) and in the sufficiently exact numerical solution of the difference equations following from expansion of the wave function over finite basis (Benassi ans Grecchi 1980, Maquet et al 1983, Kolosov 1987, Telnov 1989, AnokhinIvanov 1994), complex-coordinate method, quantum defect approximation etc [20-39]. Hehenberger, McIntosh and E. Brändas have applied the Weyl's theory to the Stark effect 
in the hydrogen atom. They have shown that one of the interesting features of Weyl's theory is that it requires a complex parameter and complex solutions to the differential equations making it a powerful tool for the treatment of resonance states. Brändas and Froelich have shown that a complex scale transformation of the time-dependent Schrödinger equation leads to a symmetric $\mathrm{EE}$ value problem containing both bound states and resonance (complex) EE values as solutions. It is worth to note that application of the complex coordinate method to a resonance problem has been justified in [20-33].

Themelis and Nicolaides [2-4] adopted an $\mathrm{ab}$ initio theory to compute the complex energy of multielectron atomic states and applied it to computing the energies and widths of the lithium Stark resonances for weak and strong fields. Their approach is based on the state-specific construction of a nonHermitian matrix according to the form of the decaying-state EF which emerges from the complex eigenvalue Schrodinger equation (CESE) theory. Jianguo Rao et al and Hui-Yan Meng et al [1] have elaborated the B-spline-based coordinate rotation method plus the model potential approach and applied it to investigate the complex energies of low-lying resonances of the hydrogen and lithium atoms in an electric field. Sahoo and Ho [5] carried out the calculation the Stark resonances energies and widths in the lithium atom on the base of the complex absorbing potential (CAP) method. It should be noted that the authors use a model potential to represent the interaction between the inner core electrons with the outside valence electron. In fact, these methods are based on the single-active-electron (SAE) approximation. In Refs.[56-58] it had been presented a consistent uniform quantum approach to the solution of the non-stationary state problems including the DC (Direct Current) strongfield Stark effect and also scattering problem. It is based on the operator form of the perturbation theory (OPT) for the Schrödinger equation. A model potential to represent the interaction between the inner core electrons with the outside valence electron is also used in application of the OPT method to alkali atoms Stark resonances.

In this work we present an advanced calculational approach to computing the Stark resonances energies and widths for the nonhydrogenic (non-H) atomic systems in a DC electric field. Our method is based on the modified OPT method and includes the physically reasonable distorted-waves approximation in the frame of the formally exact quantum-mechanical procedure. The Stark resonances energies and widths are calculated for the $4 \mathrm{f}$ lithium state and compared with the data of calculations on the basis of the alternative sophisticated approaches such as the method of complex eigenvalue Schrödinger equation by Themelis-Nicolaides, the complex absorbing potential method by Sahoo-Ho and the B-spline-based coordinate rotation method by Hui-Yan Meng et al [1-5].

\section{Operator perturbation theory for multielectron atoms in an electric field}

As the principal ideas of the approach have been presented in Ref. [17,18], here we are limited to some key elements. As usually, we start from the Dirac Hamiltonian (in relativistic units):

$$
H=\alpha p+\beta-\alpha Z / r_{i}+\sqrt{\alpha} \cdot \varepsilon \cdot z,
$$

Here a field strength intensity $\varepsilon$ is expressed in the relativistic units $\left(\varepsilon_{r e l}=\alpha^{5 / 2} \varepsilon_{\text {at.un. }} ; \alpha\right.$ is the fine structure constant). One could see that a relativistic wave function in the Hilbert space is a bi-spinor. In order to further diagonalize the Hamiltonian (1), we need to choose the correct basis of relativistic functions, in particular, by choosing the following functions as in Ref, [17-20]. The corresponding matrix elements of the total Hamiltonian will be no-zeroth only between the states with the same $M_{\mathrm{J}}$. In fact this moment is a single limitation of the whole approach. Transformation of co-ordinates in the Pauli Hamiltonian (in comparison with the Schrodinger equation Hamiltonian it contents additional potential term of a magnetic dipole 
in an external field) can be performed by the standard way. However, procedure in this case is significantly simplified. They can be expressed through the set of one-dimensional integrals, described in details in Refs. [1724]. To simplify the calculational procedure, the uniform electric field $\varepsilon$ should be substitute by the function (c.g. [17,22]:

$$
\varepsilon(\mathrm{t})=\frac{1}{t} \varepsilon\left[(t-\tau) \frac{\tau^{4}}{\tau^{4}+t^{4}}+\tau\right]
$$

with sufficiently large $\tau\left(\tau=1.5 t_{2}\right)$. The motivation of a choice of the $\varepsilon(t)$ and some physical features of electron motion are presented in Refs. [17-20]. Here we only underline that the function $\varepsilon(t)$ practically coincides with the constant $\varepsilon$ in the inner barrier motion region, i.e. $t<t_{2}$ and disappears at $t>t_{2}$. It is important that the final results do not depend on the parameter $\tau$. It is carefully checked in the numerical calculation.

As usually (see [17-24]), the scattering states energy spectrum now spreads over the range $(-\varepsilon \tau / 2,+\infty)$, compared with $(-\infty,+\infty)$ in the uniform field. In contrast to the case of a free atom in scattering states in the presence of the uniform electric field remain quantified at any energy $E$, i.e. only definite values of $\beta_{1}$ are possible. The latter are determined by the confinement condition for the motion along the $\eta$-axis.

The same is true in our case, but only for the following interval:

$$
E \subset\left(-\frac{1}{2} \varepsilon \tau,+\frac{1}{2} \varepsilon \tau\right) .
$$

Ultimately, such a procedure provides construction of realistic functions of the bound and scattering states. In a certain sense, this completely corresponds to the advantages of the distorted-wave approximation known in scattering theory [18].

The total Hamiltonian does not possess the bound stationary states. According to Ref. [17-22], one has to define the zero order Hamiltonian $H_{0}$, so that its spectrum repro- duces qualitatively that of the initial one. To calculate the width $\Gamma$ of the concrete quasistationary state in the lowest PT order one needs only two zeroth-order EF of $H_{0}$ : bound state function $\Psi_{E b}$ and scattering state function $\Psi_{E s}$. There can be solved a more general problem: a construction of the bound state function along with its complete orthogonal complementary of scattering functions $\Psi_{E}$ with

$$
\mathrm{E} \subset\left(-\frac{1}{2} \varepsilon \tau,+\infty\right) .
$$

The imaginary part of state energy (the resonance width) in the lowest PT order is determined by the standard way:

$$
\operatorname{Im} E=\Gamma / 2=\pi\left|<\Psi_{E b}\right| H\left|\Psi_{E s}>\right|^{2}
$$

with the total Hamiltonian $H$.

The state functions $\Psi_{E b}$ and $\Psi_{E S}$ are assumed to be normalized to 1 and by the $\delta\left(k-k^{\prime}\right)$ condition, accordingly. The matrix elements $\left\langle\Psi_{E b}|H| \Psi_{E^{\prime} s}\right\rangle$ entering the high-order PT corrections can be determined in the same way. It is important to underline that These corrections can be expressed through the set of one-dimensional integrals, described in details in Refs. [17-20].

Further the ROPT scheme is combined with the RMBPT in spherical coordinates for a free atom. The details of this procedure can be found in the references [22-24]. The RMBPT formalism is constructed by the following way]. An atomic multielectron system is usually described by the relativistic Dirac Hamiltonian (the atomic units are used) as follows:

$$
\begin{aligned}
& H=\sum_{i}\left\{\alpha c p_{i}-\beta c^{2}-Z / r_{i}\right\}+ \\
& +\sum_{i>j} \exp \left(i|\omega| r_{i j}\right)\left(1-\alpha_{i} \alpha_{j}\right) / r_{i j}
\end{aligned}
$$

where $Z$ is a charge of nucleus, $\alpha_{i}, \alpha_{j}$ are the Dirac matrices, $\omega_{i j}$ is the transition frequency, $\mathrm{c}-$ the velocity of light. The interelectron interaction potential (second term in (4)) takes into account the retarding effect and magnetic interaction in the lowest order on 
parameter of the fine structure constant. In the PT zeroth approximation it is used $a b$ initio mean-field potential:

$$
V^{D K S}(r)=\left[V_{\text {Coul }}^{D}(r)+V_{X}(r)+V_{C}(r \mid a)\right],
$$

with the standard Coulomb (or some model potential analog), exchange Kohn-Sham $V_{X}$ and correlation $V c$ potentials (look details in Refs. [19,20]). An effective approach to accounting the multi-electron polarization contributions is described earlier and based on using the effective two-QP polarizable operator, which is included into the PT first order matrix elements.

In order to calculate the decay (transition) probabilities and widths an effective relativistic energy approach (version [5,6,40-43]) is used. In particular, a width of the state, connected with an autoionization decay, is determined by a coupling with the continuum states and calculated as square of the matrix element [19]:

$$
\begin{gathered}
V_{\beta_{1} \beta_{2} ; \beta_{4} \beta_{3}=\sqrt{\left(2 j_{1}+1\right)\left(2 j_{2}+1\right)\left(2 j_{3}+1\right)\left(2 j_{4}+1\right)}} \\
(-1)^{j_{1}+j_{2}+j_{3}+j_{4}+m_{1}+m_{2}} \mathrm{x} \\
\mathrm{x} \sum_{a \mu}(-1)^{\mu}\left(\begin{array}{rrr}
j_{1} & j_{3} & a \\
m_{1}-m_{3} & \mu
\end{array}\right)\left(\begin{array}{rrr}
j_{2} & j_{4} & a \\
m_{2}-m_{4} & \mu
\end{array}\right) \times \\
\mathrm{x} Q_{a}\left(n_{1} l_{1} j_{1} n_{2} l_{2} j_{2} ; n_{4} l_{4} j_{4} n_{3} l_{3} j_{3}\right)
\end{gathered}
$$

Here $Q_{a}=Q_{a}^{\mathrm{Qul}}+Q_{a}^{\mathrm{Br}}$, where $Q_{a}^{\mathrm{Qul}}$, and $Q_{a}^{\mathrm{Br}}$ correspond to the Coulomb and Breit parts of the interelectron potential and express through Slater-like radial integrals and standard angle coefficients. Other details can be found in Refs. [5,6,40-43].

The most complicated problem of the relativistic PT computing the complex multielectron elements spectra is in an accurate, precise accounting for the multi-electron exchange-correlation effects (including polarization and screening effects, a continuum pressure etc), which can be treated as the effects of the PT second and higher orders. The detailed description of the polarization dia- grams and the corresponding analytical expressions for matrix elements of the polarization QPs interaction (through the polarizable core) potential is presented in Refs. [5,6,4052].

\section{Results and Conclusions}

Here we present the results of computing the complex energy eigenvalues representing the shifted and broadened $4 \mathrm{~s}$ state of lithium atom as a function of electric field strength. Meng et al [1] have presented the similar results using an advanced B-spline based coordinate rotation (B-CR) approach plus the model potential method. Themelis and Nicolaides [4] adopted ab initio theory to compute the complex energy of multielectron states for atom in an electric field. Their approach is based on the state-specific construction of a non-Hermitian matrix according to the form of the decaying-state eigenfunction which emerges from the CESE theory. Sahoo and Ho [5] performed the calculation of the Stark resonances energies and widths on the basis of a complex absorbing potential (CAP) method.

In Table 1 we present our data on the EE representing the shifted and broadened $4 \mathrm{~s}$ state of lithium atom as a function of electric field strength (in a.u.). For comparison the analogous results, obtained on the basis of the CAP, CESE, B-CR methods [1-5] as well as semiclassical (SC) estimates, are listed too. Analysis of the results shows that our data on the Stark resonances parameters are in a physically reasonable agreement with theoretical data obtained by other, in particular, CESE and B-CR methods.

However, the results for the $4 \mathrm{f}$ lithium state width differ more significantly from each other. For example, the CAP calculation for the width of the 4f state gives systematically less values than obtained by the CESE, B-CR and our methods.

Our resonance width values are higher As it was indicated in Ref. [4], one of the advantages of the B-CR method is possibility to apply in the case of increasing field strengths without a significant computational effort 
growth, however, the convergence of the width $\Gamma$ to obtain reliable complex eigenvalues should be carefully carried out.

Table 1. Complex eigenvalues (in atomic units: a.u.) representing the shifted and broadened $4 \mathrm{f}$ state of lithium atom as a function of the field strength $\varepsilon^{*}$ (in $10^{-4}$ a.u.), calculated by different methods (see text)

\begin{tabular}{|l|c|c|c|c|}
\hline $\begin{array}{l}\mathrm{Li} \\
\text { 4f }\end{array}$ & $\begin{array}{c}\mathrm{B}-\mathrm{CR} \\
{[1]}\end{array}$ & $\begin{array}{c}\mathrm{B}-\mathrm{CR} \\
{[1]}\end{array}$ & $\begin{array}{c}\mathrm{CAP} \\
{[5]}\end{array}$ & $\begin{array}{c}\mathrm{CAP} \\
{[5]}\end{array}$ \\
\hline$\varepsilon^{*}$ & $-E_{r}$ & $\Gamma / 2$ & $-E_{r}$ & $\Gamma / 2$ \\
\hline 2.0 & $2.8962[-2]$ & $2.36[-8]$ & $2.896[-2]$ & $1.62[-7]$ \\
\hline 2.5 & $2.9303[-2]$ & $3.170[-4]$ & $2.834[-2]$ & $1.01[-4]$ \\
\hline 3.0 & $3.1036[-2]$ & $9.363[-4]$ & $2.796[-2]$ & $1.76[-4]$ \\
\hline 4.0 & $3.4574[-2]$ & $2.385[-3]$ & $2.657[-2]$ & $7.05[-4]$ \\
\hline 4.5 & $3.6162[-2]$ & $3.038[-3]$ & - & - \\
\hline 5.0 & $3.8008[-2]$ & $3.767[-3]$ & $2.576[-2]$ & $1.51[-3]$ \\
\hline 6.0 & $4.1282[-2]$ & $5.929[-3]$ & - & - \\
\hline 7.0 & $4.4043[-2]$ & $8.095[-3]$ & - & - \\
\hline 8.0 & $4.6559[-2]$ & $1.020[-2]$ & - & - \\
\hline 10 & $5.1122[-2]$ & $1.424[-2]$ & - & - \\
\hline 12 & $5.5320[-2]$ & $1.805[-2]$ & - & - \\
\hline $\mathrm{Li}$ & $\mathrm{This}$ & $\mathrm{This}$ & $\mathrm{CESE}[4]$ & $\mathrm{SC}[4]$ \\
\hline$\varepsilon^{*}$ & $-E_{r}$ & $\Gamma / 2$ & $-E_{r}$ & $\Gamma / 2$ \\
\hline 2.0 & $2.8962[-2]$ & $3.401[-8]$ & $3.445[-8]$ & $1.67[-10]$ \\
\hline 2.5 & $2.9295[-2]$ & $3.172[-4]$ & $3.172[-4]$ & $1.17[-6]$ \\
\hline 3.0 & $3.1028[-2]$ & $9.423[-4]$ & $9.482[-4]$ & $3.38[-4]$ \\
\hline 4.0 & $3.4565[-2]$ & $2.386[-3]$ & $2.386[-3]$ & 0.2654 \\
\hline 4.5 & $3.6153[-2]$ & $3.042[-3]$ & $3.049[-3]$ & - \\
\hline 5.0 & $3.7998[-2]$ & $3.806[-3]$ & $3.839[-3]$ & - \\
\hline 6.0 & $4.1273[-2]$ & $5.974[-3]$ & $6.011[-3]$ & - \\
\hline 7.0 & $4.4035[-2]$ & $8.133[-3]$ & $8.169[-3]$ & - \\
\hline 8.0 & $4.6550[-2]$ & $1.024[-2]$ & $1.027[-2]$ & - \\
\hline 10 & $5.1113[-2]$ & $1.426[-2]$ & $1.427[-2]$ & - \\
\hline 12 & $5.5312[-2]$ & $1.807[-2]$ & $1.809[-2]$ & - \\
\hline & & & & \\
\hline
\end{tabular}

One of the advantages of the modified OPT method is that an increasing a field strength does not lead to an increase of computational effort and there is no a convergence problem. To ensure rapid PT convergence, a physically reasonable spectrum (EE and EF) was chosen as the zero order, similar to the 'distorted waves' method. Indeed, the convergence tests confirmed this fact. The OPT approach provides not only reso- nance state function definition but also the construction of the complex EE state function along with its complete orthogonal complementary of the scattering functions.

In Refs. [51-61] the operator PT method ideology has been used to consider a problem of resonances in the heavy ions collisions and AC Stark effect as well as the actual problems of a cooperative combined electrongamma-nuclear spectroscopy. In any case development of advanced computational methods to Stark resonances in atoms is of a great importance for multiple physical applications [51-61].

\section{References}

1. Meng, H.-Y.; Zhang, Y.-X.; Kang, S.; Shi, T.-Y.; Zhan, M.-S. Theoretical complex Stark energies of lithium by a complex scaling plus the B-spline approach. J. Phys. B: At. Mol. Opt. Phys. 2008, 41, 155003.

2. Themelis S I and Nicolaides C. A. , Complex energies and the polyelectronic Stark problem. J. Phys. B: At. Mol. Opt. Phys. 2000, 33, 5561

3. Themelis S I and Nicolaides C A Complex energies and the polyelectronic Stark problem: II. The $\mathrm{Li} \mathrm{n}=4$ levels for weak and strong fields. J. Phys. B: At. Mol. Opt. Phys. 2001, 34, 2905

4. Mercouris T and Nikolaides C A Solution of the many-electron many-photon problem for strong fields: Application to $\mathrm{Li}-$ in one- and two-color laser fields 2003 Phys. Rev. A. 2003, 67, 063403

5. Glushkov, A.V. Relativistic Quantum theory. Quantum mechanics of atomic systems. Astroprint: Odessa, 2008.

6. Khetselius, O.Yu. Quantum structure of electroweak interaction in heavy finite Fermi-systems. Astroprint: Odessa, 2011.

7. Sahoo S and Ho Y K Stark effect on the low-lying excited states of the hydrogen and the lithium atoms. J. Phys. B: At. Mol. Opt. Phys. 2000, 33, 5151

8. Glushkov, A., Buyadzhi, V., Kvasikova, A., Ignatenko, A., Kuznetsova, A., Prepelitsa, G., Ternovsky, V. Non-Linear chaotic dynamics of quantum systems: 
Molecules in an electromagnetic field and laser systems. In: Quantum Systems in Physics, Chemistry, and Biology. Springer, Cham. 2017, 30, 169-180.

9. Harmin, D.A. Theory of the Stark effect. Phys. Rev. A 1982, 26, 2656.

10.Popov, V.; Mur, V.; Sergeev A.; Weinberg, V. Strong-field Stark effect: perturbation theory and $1 / \mathrm{n}$ expansion. Phys. Lett. A 1990, 149(9), 418-424.

11. Glushkov, A.V.; Ambrosov, S.V.; Ignatenko, A.V. Non-hydrogenic atoms and Wannier-Mott excitons in a DC electric field: Photoionization, Stark effect, Resonances in ionization continuum and stochasticity. Photoelectr. 2001, 10, 103106.

12. Glushkov, A.V. Spectroscopy of atom and nucleus in a strong laser field: Stark effect and multiphoton resonances. $J$. Phys.: Conf. Ser. 2014, 548, 012020

13. Glushkov A. Spectroscopy of cooperative muon- gamma- nuclear processes: Energy and spectral parameters. J. Phys.: Conf. Ser. 2012, 397, 012011

14. Ignatenko, A.V. Probabilities of the radiative transitions between Stark sublevels in spectrum of atom in an DC electric field: New approach. Photoelectronics, 2007, 16, 71-74.

15. Glushkov, A.; Ambrosov, S.; Ignatenko, A. Non-hydrogenic atoms and WannierMott excitons in a DC electric field: Photoionization, Stark effect, Resonances in ionization continuum and stochasticity. Photoelectronics, 2001, 10, 103-106.

16. Glushkov, A.V.; Ternovsky, V.B.; Buyadzhi, V.; Prepelitsa, G.P. Geometry of a Relativistic Quantum Chaos: New approach to dynamics of quantum systems in electromagnetic field and uniformity and charm of a chaos. Proc. Intern. Geom. Center. 2014, 7(4), 60-71.

17. Glushkov A.V.; Ivanov, L.N. DC strongfield Stark effect: consistent quantummechanical approach. J. Phys. B: At. Mol. Opt. Phys. 1993, 26, L379-386

18. Glushkov, A.V. Operator Perturbation Theory for Atomic Systems in a Strong DC Electric Field. In: Hotokka M.,
Brändas E., Maruani J., Delgado-Barrio G. (eds) Advances in Quantum Methods and Applications in Chemistry, Physics, and Biology.Eds.; Springer: Cham. 2013, 27, 161-177.

19. Glushkov, A. Atom in an electromagnetic field. KNT: Kiev, 2005.

20. Glushkov, A.V. Relativistic and correlation effects in spectra of atomic systems; Astroprint: Odessa, 2006.

21. Khetselius, O.Yu. Hyperfine structure of atomic spectra. Astroprint: Odessa, 2008

22. Kuznetsova, A.A.; Glushkov, A.V.; Ignatenko, A.V.; Svinarenko, A.A.; Ternovsky V.B. Spectroscopy of multielectron atomic systems in a DC electric field. Adv. Quant. Chem. (Elsevier) 2018, 78, 287-306.

23. Kuznetsova, A.; Buyadzhi, A.; Gurskaya, M.; Makarova, A. Spectroscopy of multi electron atom in a DC electric field: Modified operator perturbation theory approach to Stark resonances. Photoelectronics. 2018, 27, 94-102

24. Kuznetsova, A.A.; Glushkov, A.V.; Romanenko, E.S.; Plisetskaya, E.K., Spectroscopy of multielectron atom in dc electric field: relativistic operator perturbation theory. Photoelectronics. 2019, 28, 32-38

25. Froelich, P.; Davidson, E.R.; Brändas, E. Error estimates for complex eigenvalues of dilated Schr6dinger operators. Phys. Rev. A 1983, 28, No. 5, 2641.

26. Lipkin, N.; Moiseyev, N.; Brändas, E. Resonances by the exterior-scaling method within the framework of the finitebasis-set approximation. Phys. Rev. A 1989, 40, No. 2, 549.

27. Brändas, E.; Froelich, P.; Obcemea, C.H., Elander, N.; Rittby, M. Note on the complex stabilization method. Phys. Rev. A 1982, 26, No. 6, 3656.

28. Resonances. The Unifying Route Towards the Formulation of Dynamical Processes - Foundations and Applications in Nuclear, Atomic and Molecular Physics, Series: Lecture Notes in Physics; Brändas, E.; Elander, N. Eds.; Springer: Berlin, 1989, 325, 1-564. 
29. Ostrovsky, V.N.; Elander, N. Scattering resonances and background associated with an asymmetric potential barrier via Siegert pseudostates. Phys. Rev. A 2005, 71, 052707.

30. Sigal, I. Geometric theory of Stark resonances in multielectron systems. Comm. Math. Phys. 1988, 119, 287-314.

31. Silverstone, H.J.; Adams, B.G.; Cizek, J.; Otto, P. Stark Effect in Hydrogen: Dispersion Relation, Asymptotic Formulas, and Calculation of the Ioni-zation Rate via High-Order Perturbation Theory. Phys. Rev. Lett. 1979, 43, No. 20, 14981501.

32. Cerjan, C.; Hedges, R.; Holt, C.; Reinhardt, W.P.; Scheibner, K.; Wendoloski, J.J. Complex coordinates and the Stark effect. Int. J. Quant. Chem. 1978, 14 (4), 393-418.

33. Luc-Koenig, E.; Bachelier, A. Systematic theoretical study of the Stark spectrum of atomic hydrogen. I. Density of continuum states. J. Phys. B: At. Mol. Phys. 1980, 13, 1743-1756

34. Damburg, R.J.; Kolosov, V.V. A hydrogen atom in a uniform electric field. $J$. Phys. B: At. Mol. Phys. 1976, 9, No. 18, 3149.

35. Maquet, A.; Chu, S.I.; Reinhardt, W.P. Stark ionization in dc and ac fields: An L2 complex-coordinate approach. Phys. Rev. A 1983, 27, No. 6, 2946-2970.

36. Reinhardt, W.P. Padé summations for the real and imaginary parts of atomic stark eigenvalues. Int. J. Quant. Chem. 1982, 21(1), 133-146.

37. Franceschini, V.; Grecchi, V.; Silverstone, H. Complex energies from real perturbation series for the LoSurdo-Stark effect in hydrogen by Borel-Padé approximants. J. Phys. Rev. A 1985, 32 (3), 1338.

38. Telnov, D.A. DC Stark effect in a hydrogen atom via Sturmian expansions. $J$. Phys. B.: At. Mol. Opt. Phys. 1989, 22, No. 14, L399-403.

39. Ivanov, I.; Ho, Y.-K. Complex rotation method for the Dirac Hamiltonian. Phys. Rev. A 2004, 69, 023407.
40. Ivanov, L.N.; Ivanova, E.P. Method of Sturm orbitals in calculation of physical characteristics of radiation from atoms and ions. JETP. 1996, 83, 258-266.

41. Ivanova, E.P.; Ivanov, L.N.; Glushkov, A.V.; Kramida, A.E. High order corrections in the relativistic perturbation theory with the model zeroth approximation, Mg-Like and Ne-Like Ions. Phys. Scripta 1985, 32, 513-522.

42. Ivanova, E.P.; Glushkov, A.V. Theoretical investigation of spectra of multicharged ions of F-like and Ne-like isoelectronic sequences. J. Quant. Spectr. Rad. Transfer. 1986, 36, 127-145.

43. Glushkov, A.V. Advanced relativistic energy approach to radiative decay processes in multielectron atoms and multicharged ions. In Quantum Systems in Chemistry and Physics: Progress in Methods and Applications. Springer: Dordrecht, 2012; Vol. 26, pp 231-252.

44. Svinarenko A., Glushkov, A., Khetselius, O., Ternovsky,V., Dubrovskaya, Yu., Kuznetsova, A., Buyadzhi, V. Theoretical spectroscopy of rare-earth elements: spectra and autoionization resonances. Rare Earth Element. InTech, 2017, pp 83-104.

45. Glushkov, A.V., Khetselius, O.Yu., Svinarenko, A.A., Buyadzhi, V.V., Spectroscopy of autoionization states of heavy atoms and multiply charged ions. TEC: Odessa, 2015.

46. Glushkov, A.V., Khetselius, O.Yu., Svinarenko, A., Buyadzhi, V. Methods of computational mathematics and mathematical physics. P.1. Odessa: 2015.

47. Khetselius, O., Glushkov, A., Dubrovskaya, Yu., Chernyakova, Yu., Ignatenko, A.V., Serga, I., Vitavetskaya, L. Relativistic quantum chemistry and spectroscopy of exotic atomic systems with accounting for strong interaction effects. In: Concepts, Methods and Applications of Quantum Systems in Chem. and Phys. Springer, Cham, 2018, 31, 71-91.

48. Glushkov, A.V., Khetselius, O.Yu., Svinarenko A.A., Buyadzhi, V.V., Ternovsky, V.B, Kuznetsova, A., Bashkarev, 
P Relativistic perturbation theory formalism to computing spectra and radiation characteristics: application to heavy element. Recent Studies in Perturbation Theory, ed. D. Uzunov (InTech) 2017, 131-150.

49. Khetselius, O. Relativistic perturbation theory calculation of the hyperfine structure parameters for some heavy-element isotopes. Int. Journ. Quant. Chem. 2009, 109, 3330-3335.

50. Khetselius, O. Relativistic calculation of the hyperfine structure parameters for heavy elements and laser detection of the heavy isotopes. Phys. Scr. 2009, 135, 014023

51. Khetselius, O.Yu., Spectroscopy of cooperative electron-gamma-nuclear processes in heavy atoms: NEET effect. $J$. Phys.: Conf. Ser. 2012, 397, 012012.

52. Svinarenko, A. Study of spectra for lanthanides atoms with relativistic manybody perturbation theory: Rydberg resonances. J. Phys.: Conf. Ser. 2014, 548, 012039.

53. Buyadzhi, V., Zaichko, P., Antoshkina, O., Kulakli, T., Prepelitsa, G., Ternovsky, V., Mansarliysky, V. Computing of radiation parameters for atoms and multicharged ions within relativistic energy approach: Advanced Code. J. Phys.: Conf. Ser. 2017, 905(1), 012003.

54.Buyadzhi, V.V., Glushkov, A.V., Mansarliysky, V.F., Ignatenko, A.V., Svinarenko, A.A. Spectroscopy of atoms in a strong laser field: new method to sensing ac stark effect, multiphoton resonances parameters and ionization cross-sections. Sensor Electr. and Microsyst. Techn. 2015, 12(4), 27-36.
55. Ambrosov, S., Khetselius, O., Ignatenko, A. Wannier-Mott exciton and $\mathrm{H}, \mathrm{Rb}$ atom in a DC electric field: Stark effect. Photoelectronics. 2008, 17, 84-87.

56. Ambrosov S., Ignatenko V., Korchevsky D., Kozlovskaya V. Sensing stochasticity of atomic systems in crossed electric and magnetic fields by analysis of level statistics for continuous energy spectra. Sensor Electr. and Microsyst. Techn. 2005, Issue 2, 19-23.

57. Buyadzhi, V.V. Laser multiphoton spectroscopy of atom embedded in Debye plasmas: multiphoton resonances and transitions. Photoelectrs. 2015, 24, 128.

58. Buyadzhi, V.V.; Chernyakova, Yu.G.; Smirnov, A.V.; Tkach, T.B. Electroncollisional spectroscopy of atoms and ions in plasma: Be-like ions. Photoelectronics. 2016, 25, 97-101.

59. Buyadzhi, V.; Chernyakova, Yu.; Antoshkina, O.; Tkach, T. Spectroscopy of multicharged ions in plasmas: Oscillator strengths of Be-like ion Fe. Photoelectronics. 2017, 26, 94-102.

60. Chernyakova, Y.G., Ignatenko A.V., Vitavetskaya L.A. Sensing the tokamak plasma parameters by means high resolution x-ray theoretical spectroscopy method: new scheme. Sensor Electr. and Microsyst. Techn. 2004, 1, 20-24

61. Khetselius, O.Yu., Gurnitskaya, E.P., Loboda, A.V., Vitavetskaya, L.A. Consistent quantum approach to quarkony energy spectrum and semiconductor superatom and in external electric field. Photoelectronics. 2008, 17, 127-130. 


\section{THEORETICAL COMPLEX ENERGIES OF STARK RESONANCES IN LITHIUM BY OPERATOR PERTURBATION THEORY APPROACH}

Summary. The theoretical complex energies of the Stark resonances in the lithium atom (non-hydrogenic atomic system) in a DC electric are calculated within the operator form of the modified perturbation theory of the Schrödinger equation for the non- $\mathrm{H}$ atomic systems. The method includes the physically reasonable distorted-waves approximation in the frame of the formally exact quantum-mechanical procedure. The Stark resonances energies and widths in the lithium atom spectrum are calculated and compared with results of calculations on the basis of the complex absorbing potential method, the B-spline-based coordinate rotation method approach and direct computing complex Schrödinger equation eigenvalues.

Keywords: multielectron atom, electric field, relativistic operator perturbation theory, excited states.

PACS 31.15.A-

Кузнецова А.А., Глушков А.В., Плисечкая Е.К.

\section{ТЕОРЕТИЧЕСКИЕ ЗНАЧЕНИЯ КОМПЛЕКСНЫХ ЭНЕРГИЙ ШТАРКОВСКИХ РЕЗОНАНСОВ В АТОМЕ ЛИТИЯ В РАМКАХ ОПЕРАТОРНОЙ ТЕОРИИ ВОЗМУЩЕНИЙ}

Резюме. Вычислены значения комплексных энергий штарковских резонансов в атоме лития (многоэлектронная атомная система) в постоянном электрическом поле на основе модифицированной операторной теории возмущений для многоэлектронных атомных систем. Теоретический подход включает физически обоснованное приближение искаженных волн в рамках формально точной квантово-механической процедуры. Энергии и ширины штарковских резонансов в спектре атома лития вычисляются и сравниваются с результатами расчетов в рамках метода комплексного оптического потенциала, обобщенного метода вращения координат с использованием В сплайнового алгоритма и данными прямого вычисления собственных значений комплексного уравнения Шредингера.

Ключевые слова: Многоэлектронные атом, электрическое поле, релятивистская операторная теория возмущений оператора, возбужденные состояния 
Кузнецова Г.О., Глушков О.В., Плісецькка С.К.

\section{ТЕОРЕТИЧНІ ЗНАЧЕННЯ КОМПЛЕКСНИХ ЕНЕРГІЙ ШТАРКІВСЬКИХ РЕ- ЗОНАНСІВ В АТОМІ ЛІТІЮ В РАМКАХ ОПЕРАТОРНОЇ ТЕОРІЇ ЗБУРЕНЬ}

Резюме. Обчислені значення комплексних енергій штарківських резонансів в атомі літію (багатоелектронних атомна система) в постійному електричному полі на основі модифікованої операторної теорії збурень для багатоелектронних атомних систем. Теоретичний підхід включає фізично обгрунтоване наближення перекручених хвиль в рамках формально точної квантово-механічної процедури. Енергії і ширини штарківських резонансів в спектрі атома літію обчислюються і порівнюються з результатами розрахунків в рамках методу комплексного оптичного потенціалу, узагальненого методу обертання координат з використанням В-сплайнового алгоритму і даними прямого обчислення власних значень комплексного рівняння Шредінгера.

Ключові слова: багатоелектронний атом, електричне поле, релятивістська операторна теорія збурень оператора, збуджені стани. 